\title{
Perspectivas 2001
}

\begin{abstract}
Las expectativas al inicio del año antes de los terremotos eran positivas bajo la óptica gubernamental. A juicio de las autoridades gubernamentales existian factores externos e internos, que conjugados imprimian optimismo a las mismas para proyectar una reactivación de la economia, a pesar de que el año 2000, se caracterizó por un lento crecimiento y un mayor desempleo, y de que la estabilidad monetaria se mantuvo divorciada de una corriente real en muchos aspectos estancada.
\end{abstract}

$L$ os factores externos mencionados se identifican con la ampliación de la iniciativa de la Cuenca del Caribe (ICC) y la implementación del Tratado del Libre Comercio con México, que se asume, abrirán espacios para más exportaciones y una mayor demanda interna. Una desaceleración de la economía estadounidense ya era contemplada en tal escenario, como un elemento de contrapeso en los potenciales ingresos de divisas, tanto en concepto de ventas de bienes, como de remesas familiares.

Una perspectiva gubernamental de un 4.5 por ciento, de crecimiento del Producto Interno Bruto (PIB) real era la de antes de los terremotos ocurridos en los primeros dos meses del presente año. Para el Banco Central de Reserva (BCR) y otros voceros de la política económica oficial al parecer, ese crecimiento es factible aún en las circunstancias presentes; posiblemente la tesis se fundamente en la convicción de que una inversión pública masiva de reconstrucción estimule la inversión y el consumo privado, en armonía con los preceptos de la teoría keynesiana de utilizar a la inversión pública como motor para alentar la demanda efecriva. Sin duda alguna, la teoría keynesiana ha sido duramente cuestionada por los defensores del sistema de mercado, pero parece que en las actuales circunstancias económicas del país adquiere validez frente a un sector empresarial que difícilmente respondería al esfuerzo de la reconstrucción.

Existen dudas respecto a lo anterior por las siguientes circunstancias: primero, el financiamiento y/o donación externa pueden resultar insuficientes; segundo, la falta de capacidad de respuesta del gobierno por razones operativas $y$ financieras; $y$ tercero, el daño en la planta productiva agrícola, industrial y del comercio con repercusiones en el PIB a corto plazo de esos sectores. No se descarta una generación de empleo importante como consecuencia de los programas de reconstrucción que obviamente permitirán un crecimiento, pero de menor cuantía al previsto originalmente por las autoridades. La inflación pudiera verse alterada hacia el alza por un exceso de demanda momentáneo. Estimaciones de la Universidad Tecnológica de El Salvador (UTEC) prevén un incremento moderado del PIB no mayor del 2 por ciento, un incremento del déficit fiscal cercano al 4 por ciento del PIB y una tasa de inflación no menor del 5 por ciento. Las remesas familiares seguirán jugando un papel providencial en el nivel de las reservas internacionales netas.

Un aspecto que merece un comentario especial es la importancia que han tenido las remesas familiares, no sólo en la acumulación de las reservas internacionales, sino también en la determinación de la liquidez de la economía y en la cobertura de acción que les proporciona esos fondos a las autoridades respectivas para sostener la estabilidad monetaria. En tal sentido la coyuntura favorable que ha venido

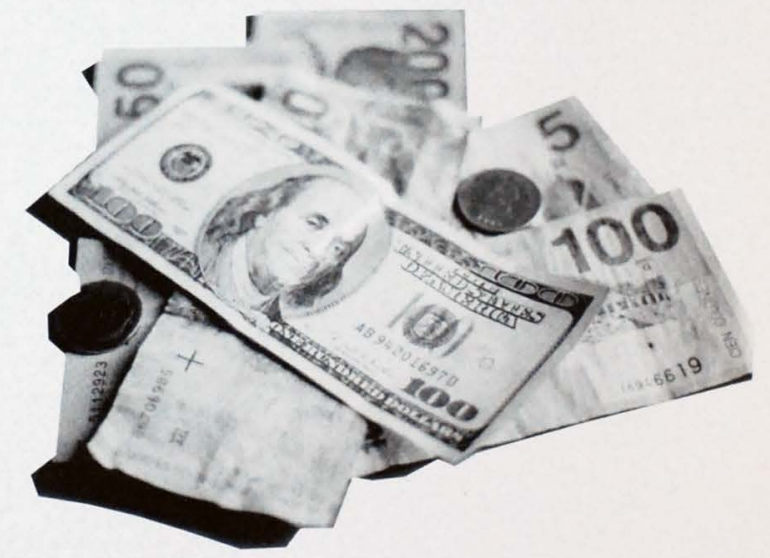


experimentando la economía estadounidense en los últimos años y que pareciera que tiende a debilitarse desde mediados del 2000, cobra especial relevancia en las perspectivas económicas de El Salvador para el 2001, precisamente por esa cada vez más estrecha dependencia de los ingresos de divisas por la venta de bienes y servicios a los Estados Unidos.

La Comisión Económica para América Latina y el Caribe (CEPAL) en un informe reciente asegura que la economía decrecerá siete o más años, lo que se interpreta que se necesitará ese lapso de tiempo para reponer el nivel de producción per cápita anterior o los niveles acumulados de formación de capital. La CEPAL también hace alusión a un porcentaje del daño con respecto a las exportaciones, a las importaciones y a la formación bruta de capital fijo. De los datos proporcionados por este organismo internacional se desprende que los terremotos afectaron considerablemente los sectores de Vivienda, Transporte, Educación y las actividades productivas de pequeños y medianos comerciantes, empresarios y artesanos. La comisión estimó en más de mil 600 millones de dólares, los daños causados por los sismos del 13 de enero $y$ el 13 de febrero, haciendo la salvedad que los estimados no incluyen todos los daños, los cuales podrían ser precisados cuando se realicen los estudios pertinentes. En general el análisis de la CEPALubica las proyecciones de crecimiento entre el 3 y el 5 por ciento para el 2001, la inflación la estima entre el 3 y el 4.3 por ciento; el déficit fiscal rondaría del 2.7 al 5 por ciento; el déficit en cuenta corriente entre el 2.5 y el 4 por ciento del PIB y la deuda pública del 32 al 35 por ciento del PIB.

La Fundación Salvadoreña para el Desarrollo Económico y Social (FUSADES) considera que la economía crecerá en el 2001 entre 2 y 2.5 por ciento; el déficit podría llegar a 3.7 por ciento; la inflación promedio 3 por ciento; el déficit comercial 16.3 por ciento y el déficit en cuenta corriente 2.1 por ciento; todos los porcentajes con referencia o relación al PIB.

Estas estimaciones son una aproximación hacia una posible realidad para el final del año 2001, pero en las actuales circunstancias, toda estimación y todo posible escenario está sujeto a erráticos comportamientos que exigen una detenida construcción de esos escenarios y de un seguimiento con- tinuo de las variables claves de la oferta y demanda globales, pues la incertidumbre de muchos factores endógenos en juego restan credibilidad a posibles comportamientos económicos, propios de una situación de desastre y emergencia.

Tomando en cuenta esa situación anómala la UTEC realizará análisis coyunturales con cierta frecuencia, a fin de generar opinión sobre el acontecer económico y las perspectivas en el mediato e inmediato plazo.

\section{Las expectativas gubernamentales} para el 2001

o único claro de la acción gubernamental es la cons-
tante intención de cerrar tratados comerciales con otros países, con el propósito de ampliar el mercado para la producción nacional. Si eso fuera asi de fácil, y con cada tratado comercial firmado existieran posibilidades concretas de ampliar el mercado a nuestros productores nada podría decirse al respecto. Pero un tratado internacional de comercio no es un acco de beneficencia para ninguna de las partes contratantes. En este sentido los demás países también esperan que el mecanismo en marcha también les proporcione una ampliación de mercado para su producción. Al final de cuentas, la balanza se inclina hacia donde se encuentran las mayores capacidades de competencia y los menores costos. El Tratado de Libre Comercio con México y la ICC con los Estados Unidos de América han sido las dos opciones estratégicas de la política gubernamental en el 2000 . No es posible ignorar que ambos países, los Estados Unidos y México son economías de dimensiones significativamente grandes en relación con la nuestra y que la opción de competir no existe en términos de mercado, a excepción de tratamientos de favor.

El Tratado con México está clasificado como asimétrico, es decir hay tratamientos explícitos de favor hacia la economía más débil, y con los Estados Unidos igualmente es un acuerdo a favor de las empresas de maquila, sujeto al cumplimiento de ciertos comportamientos políticos que nada tienen que ver con las reglas del mercado. 


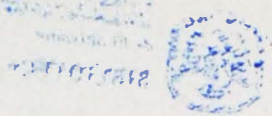

Lo preocupante es que el crecimiento de la economía nacional queda sujeto a las posibilidades que brindan paises extranjeros, dejando fuera de toda prioridad en la política el fortalecimiento del mercado nacional y la corrección de los problemas estrucrurales. Los dirigentes de la política económica cuando piensan en inversiones centran su atención en la inversión extranjera sin tener en cuenta la necesidad de promover la inversión nacional; de por sí es una politica deformante que pone en riesgo la capacidad productiva interna.

El comercioy la industria se encuentran deprimidos, las cifras del lndice de Valor Económico (IVAE) que recoge el pulso de un grupo significativo de empresas de dichos sectores muestra indiscuribles bajas; y el sector agrícola que deliberadamente fue marginado desde el primer gobierno de ARENA, resiente desde hace más de diez años de un enfoque sumamente discurible y considera que la agricultura no tiene importancia en un esquema de globalización y por lo tanto no merece de la atención de la política económica gubernamental. Para confirmar este enfoque es suficiente recordar recientes declaraciones del Presidente Francisco Flores, en donde insiste sobre la escasa perspectiva económica de los “cultivos tradicionalesn, recomendando a los productores agrícolas considerar muy detenidamente sus decisiones de continuar con dichos cultivos. Estas declaraciones, cuyo sentido aún nos inquieta, reflejan a nuestro juicio un sesgo y una inacertada visión de nuestra realidad productiva. Es cierto que los precios internacionales de los productos agrícolas se mantienen muy bajos en los mercados de los mismos, pero tradicionalmente han generado altos niveles de empleo en la población rural, empleos que no han sido cubiertos

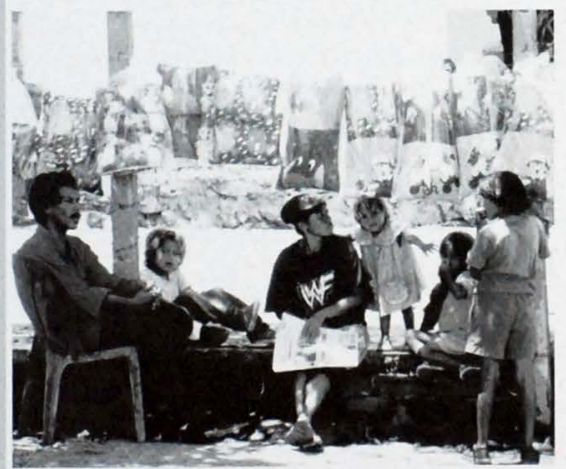

por otros sectores de la economia. Hay que buscar en este sector una parte de las explicaciones de los males de la economía.

Frente a una crisis inderenible, el gobierno se vio forzado a aceptar una recesión que hasta aquí no ha podido ser corregida por el accionar exclusivo del mercado. Los pode- rosos frentes empresariales han mostrado su descontento y han hecho veladas llamadas de atención; pero parece que las soluciones esraban entrampadas por una realidad estructural que se ha pretendido ignorar durante más de una década. Se ha pretendido culpar al incremento de los precios internacionales los problemas del año 2000, también se buscan culpables en los sectores sociales que se atreven a manifestar su descontento $y$ la oposición es responsable de las tardias aprobaciones presupuestarias y por la renuencia a aprobar nuevos préstamos externos. En resumen, el gobierno es una pobre vícrima de siniestras maquinaciones enemigas del progreso.

La dolarización de la economía fue la fórmula mágica del final del año. Una ley sorpresiva y bruscamente aprobada por la mayoria de las fuerzas políticas del país, pone en marcha, a partir de enero del 2001 , un proceso para intentar sustituir rápidamente el colón salvadoreño por el dólar norteamericano. Los partidos de oposición, y especialmente los diferentes sectores de la población salvadoreña no tuvieron opinión sobre una medida que afecta directa y sensiblemente los intereses de los diversos estratos poblacionales. Que la dolarización no es la panacea fue aceptado por el gobierno y la medida se defendió argumentando una reducción en las tasas de interés pasivas del sistema financiero. La pregunta se mantiene ¿No era posible reducir los intereses sin necesidad de cambiar el signo monetario?

\section{El comportamiento de las fuerzas sociales}

El comportamiento de las fuerzas sociales está muy difíciles los ajustes y la necesaria armonización de las distintasacciones. El choque permanente entre sectores sociales es desgastante, y al final la coincidencia de objecivos y esfuerzos para lograr la superación de la crisis no se ha producido. A nuestro juicio el gobierno debc 


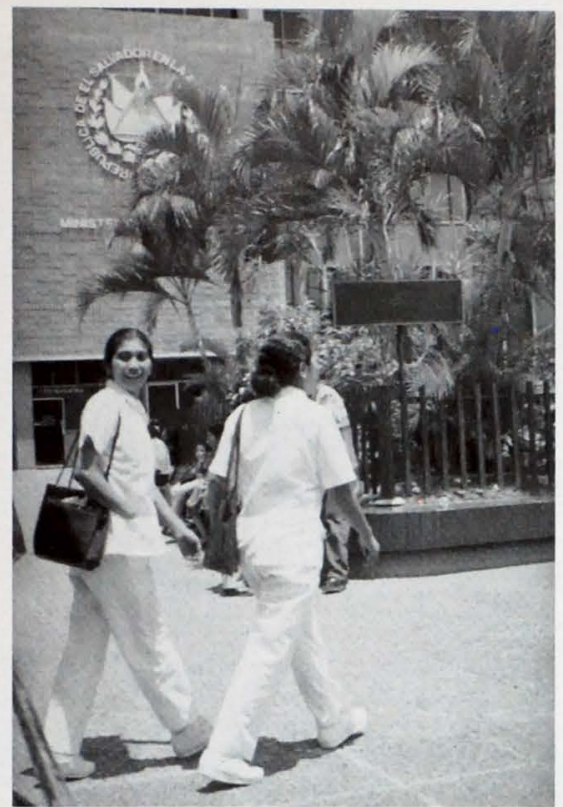

jugar un papel conciliador y de concertación, pero ha entrado a la contienda en contra de cualquier grupo social que cuestione sus enfoques y sus acciones.

Se está llegando al extremo de satanizar las opiniones adversas y se ha observado una defensa irracional frente a los errores comeridos por varios ministros. Preocupa, y es necesario apuntarlo, una subterránea tendencia dictarorial provocada por una exagerada posición de infalibilidad.

Las tensiones, normales en un proceso dinámico de cambios deberían conducir a un ambiente creativo de participación popular a favor de legítimos intereses sociales; pero el problema que se produce es que generalmente las tensiones no encuentran saliday el efecto es una postergación de la tensión o un aumento de la misma para una futura oportunidad.

Mientras las tensiones de la sociedad no encuentren salidas viables y oportunas se corre el peligro de ir hacia estallidos sociales que por regla general desbordan los mecanismos de la democracia. Como ejemplo sería suficiente seńalar el conflicto todavía no resuelto entre el gremio médico y los trabajadores de la salud con el gobierno, especificamente las autoridades del Seguro Social y el ministerio de Salud. Tambićn se cuenta las interminables conflictos entre los empresarios de buses y el gobierno, los vendedores callejeros y la municipalidad.

\section{Diferentes ópticas} a opinión pública generada por las encuestas que
algunas instituciones de prestigio realizan, con alguna sistemaricidad, son un indicador importante de los problemas sociales más relevantes y las impresiones de los ciudadanos sobre los mismos. Es duro aceptar los veredictos poblacionales, especialmente cuando se señalan fallas y errores en la gestión gubernamental. Pero hay una miopía irracional cuando las instituciones afectadas resuelven los señalamientos desmeritando los resultados de las encuestas. Esto ya se volvió un lugar común, aunque después deban rendirse ante las evidencias, cuando las votaciones revalidan los seńalamientos de las encuestas.

Para finales del año, una encuesta realizada por el Instituto Universitario de Opinión Pública (IUDOP) de la Universidad Centroamericana, publicada en La Prensa Gráfica el 14 de diciembre del 2000, arrojaba las siguientes consideraciones: el 53.4 por ciento de los salvadoreños concordaban que los principales problemas del país eran el desempleo, la delincuencia, la pobreza, la economía nacional y la inflación. La delincuencia quedaba ubicada en un segundo lugar con el 34.6 por ciento. Además el 43.9 de los encuestados cree que la economia del país empeoró, así mismo un 31.5 por ciento considera que el bienestar familiar tambićn se ha deteriorado. Las opiniones son contundentes, pese a la continua campaña triunfalista del gobierno sobre el excelente estado de la economía, la 


\section{(1)}

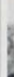

entorno entorno entorno entoring entoing entorno entoring entoring entoring entorints entorint sintorint entorint entornd entorn: entorn entornd entornt antiontint entorin entornte entoring Antortin: Entorint Antorint Entorint entorino Antoring entoritat entotit

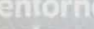
entoring snitornit) entorint entorint

entorno entorn entorn entory entor entor: entorn entorns eatroting emplco y la pobreza. manifestaciones ocurridos en $\mathrm{el}$ año tienen causas económicas, pero los voceros del gobierno sostienen que las motivaciones son políricas bajo el simple deseo de dañar la gestión gubernamental.

En lo que respecta a la confirmación de que cl cuerpo policial estaba infiltrado por delincuentes, lo que llevó a sus autoridades a poner en marcha un plan de depuración en el seno de la Policía Nacional Civil, el 64.4 por ciento cree que ésta institución ha perdido la confianza y el apoyo de la población. El 52.6 por ciento, coincide en que la delincuencia ha aumentado y el 17.3 por ciento manifiesta haber sido vícrima de una acción delincuencial. Por eso un 50.9 por ciento de la población espera que la depuración policial logre combatir la delincuencia.

El Órgano Judicial, cuya transparencia y trabajo han sido severamente cuestionados, se enfrenta a la opinión del 33 por ciento de la población encuestada, quienes consideran que la aplicación de justicia ha empeorado; pero cl 40 por ciento dice que no ha empeorado ni mejorado y solamente el 16.3 por ciento afirma la existencia de alguna mejoría.

En todas las encuestas las cifras son indicadores de los fenómenos que se miden, pero los más importantes son las tendencias de los fenómenos que se auscultan. La sociedad salvadoreña está emproblemada, a pesar de la resistencia a reconocerlo, el desempleo, la pobreza, la recesión de la economía, la delincuencia y la violencia están a la orden del día. El problema real se encuentra en que las autoridades gubernamentales no quieren reconocerlo, obviamente por intereses políticos. sin entender que en la medida que los problemas se ignoran no es posible resolverlos.

población sigue resintiendo las duras condiciones del des-

Un 53.1 por ciento de los entrevistados opinan que en el año 2000 la pobręa aumentó y solamente el 3.6 por ciento piensa que ésta disminuyó; además la mayoría coincide en que los problemas económicos desencadenan otros problemas de carácter social. Los políticos de derecha y el gobierno creen que la pobreza no genera violencia y delincuencia, sino que tales problemas se dan por las tendencias naturales de ciertos individuos hacia el delito. El 44.8 por ciento de los encuestados está convencido que las huelgas, paros y
Para finalizar este panorama, se pidió una opinión sobre la recién impuesta política de dolarizar la economía, inconsultamente llevada adelante, como una camisa de fuerza, sin importar la opinión de los af ecrados. En este punto el gobierno piensa que eso es lo adecuado y no les importó la opinión popular. Los resultados de la encuesta reflejan un 47.4 por ciento en contra de la medida, un 35.5 que la considera favorable y un 17.1 de indecisos, que seguramente no tienen los elementos de juicio necesarios para emitir una opinión. 


\section{La miopía de los políticos}

Dor regla general pareciera que nuestros politicos vi-
ven fuera de la realidad que presumen conocer, apesar de adquirir la categoría de representantes de sectores de la población que les favorecen con su confianza y despuess con su voto. Muchas de sus actuaciones están cargadas de un afán de notoriedad tan relevante como la defensa de sus intereses personales. Ninguno de los sectores sociales de nuestra sociedad ciene tan bajo perfil como las figuras políticas de muchos partidos politicos, cuya existencia es solamente explicable en función de la corrupción que pareciera ser un elemento in-

dispensable del sistema.

\section{Permanentemente} somos testigos impotentes de sus exabruptos, de sus razonamientos absurdos, de su supuesta astucia para confundir $y$ engañar a un pucblo posiblemente ingenuo, pero
El sector politico en este pais, hoy en día, es una carcomida estructura que solamente sirve para «jugar a la democracia», pero que en los hechos evidencia una abismal lejanía de los mas simples elementos que enriquecen el sistema dencia una abismal lejania de los más simples elementos que enriquecen el sistema.

Desde las úluimas elecciones, en función de sus resultados, se atisbaban dos vías para el ejercicio politico: la concertación, como instrumento capaz de fortalecer el desarrollo democrático del país, o continuar con la imposición aritmética de un esquema de poder basado en la protección de los intereses de grupos económicos influyentes, mediante la compra de voluntades o los acuerdos polícicos viciados por las concesiones y los encubrimientos mutuos. Se ha adoptado este último recurso, y el partido oficial ha logrado definir y cristalizar una dirección politica similar a la de los dos anteriores gobiernos del mismo partido.

En el mensaje de su primer año de gobierno, el Presidence fue claro $y$ enfático en garantizar que iba a atravesar el puente de la concertación con sus opositores políticos; pero todo quedó en palabras y los hechos demuestran a un aท วิo y seis meses después, que el of recimicnco fue que además no cuenta con losmedios y las formas para manifestarse. En el senode sus partidos, en sus curules, en sus altas posiciones ejecurivas, tan cerca del Olimpo pero tan lejos de las necesidades de los que ufanamente sostienen que defienden.

La superficialidad es tan grande como la soberbia de sus mentes, ereen ingenuamente que sus pensamientos $y$ aproximaciones son infalibles y que la ignorancia y la escasa visión popular son las responsables de que no se comprendan sus altas dotes de estadistas y las soluciones que brotan de sus esclarecidas mentes. El sector politico en este país. hoy en día, es una carcomida estructura que solamente sirve para "jugar a la democracia", pero que en los hechos evi- más retórico que una intención definida. Todo lo contrario, la estrategia politica muy clara del gobierno ha consistido en agrupar las fuerzas de derecha representadas en la Asamblea Legislativa, para aprobar al margen de cualquier razonable argumento, la dirección de sus perspecrivas económicas, sociales y políticas.

La concertación entre las diferentes fuerzas de nuestro pais es una de las pocas opciones posibles, pero en el ámbiro de las decisiones y los intereses políticos esta opción se ha vuclco ilusoria, sin sentido, con un claro espíritu demagógico. Las actuales tensiones se verán incrementadas por la falta de salidas, y el marco de maniobra es tan reducido que no permite soluciones permanentes de mediano plazo.

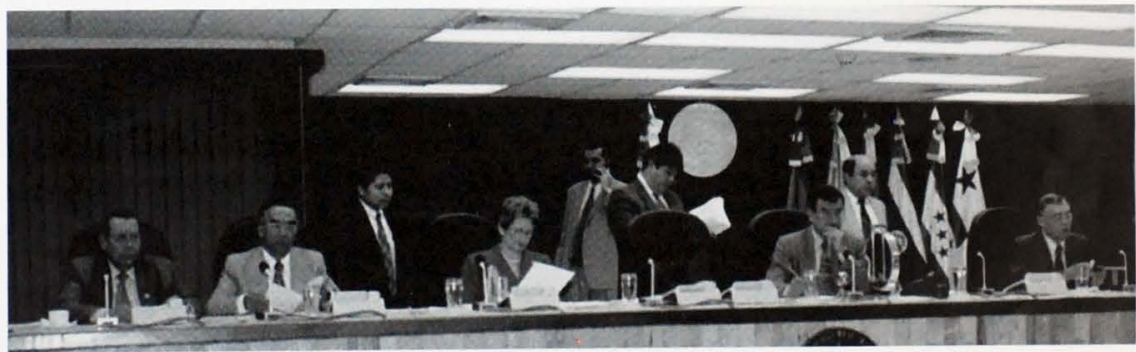




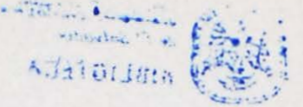

El agotamiento de la denocracia

El modelo democrático y representativo agobiado por una interminable lucha de intereses se ha desgastado aceleradamente en el escaso término de diez años. La privatización es la herramienta básica para afianzar el mercado, especialmente de sectores estratégicos que a juicio de los reformadores es necesario que estén en manos del sector privado, basados en el argumento que el Estado es mal administrador y la empresa privada es más eficiente. Es asi como preferentemente la banca, los fondos de pensiones, la electricidad y la telef onía, se subastan y venden a empresas privadas, nacionales y extranjeras. Los resultados de ésta medida son cuestionables al presente, el veredicto lo tienen los sectores populares que enfrentan incrementos en el pago de los servicios, como resultado de la ran promocionada eficiencia del sector privado.

Entonces el incipiente proyecto democrático nació desbalanceado y desde esa fecha el peso de uno solo de los sectores sociales se ha venido acentuando progresivamente. Una democracia no puede funcionar en esas condiciones y tampoco garantizar las normas equitativas de convivencia social. El proyecto de la democracia salvadoreña está siendo puesto en peligro en la medida que no puede garantizar condiciones mínimas para su desarrollo. La existencia de partidos de oposición constituyen una simple fachada, y la presencia de un partido de izquierda que nació de los Acuerdos de Paz, como representante de los sectores combatientes de la pasada guerra, no cuenta con los mecanismos para equilibrar fuerzas, y está sometido por la ilogicidad de una aritmética política a los vaivenes $\mathrm{e}$ intereses de la derecha.

Los factores que a nuestro juicio están influyendo en el agotamiento del esquema democrático son: la ausencia de reglas de juego claras entre los acrores políticos y sus relaciones dentro de los marcos de gestión del proceso, una total carencia de ética y de valores democráticos, una evidente corrupción que ha desacreditado, en la medida que los hechos se han ido evidenciando al sector político como un todo.

Como ejemplo puede señalarse la poco ćrica con que acruaron los partidos de la derecha cuando impidieron la llegada del Partido Frente Farabundo Marti para la Liberación Nacional (FMLN) a la Presidencia de la Asamblea Legislariva, el 27 de abril. Mediante una sucia alianza los partidos Alianza Republicana Nacionalista (ARENA), Conciliación Nacional (PCN) y Demócrata Cristiano (PDC), modificaron el reglamento interno de la Asamblea, para asegurarle la presidencia a uno de sus incondicionales acompañantes. De más está decir que tradicionalmente se había acordado que la presidencia correspondía al parrido con mayor cantidad de votos en las elecciones, pero indiscuti-

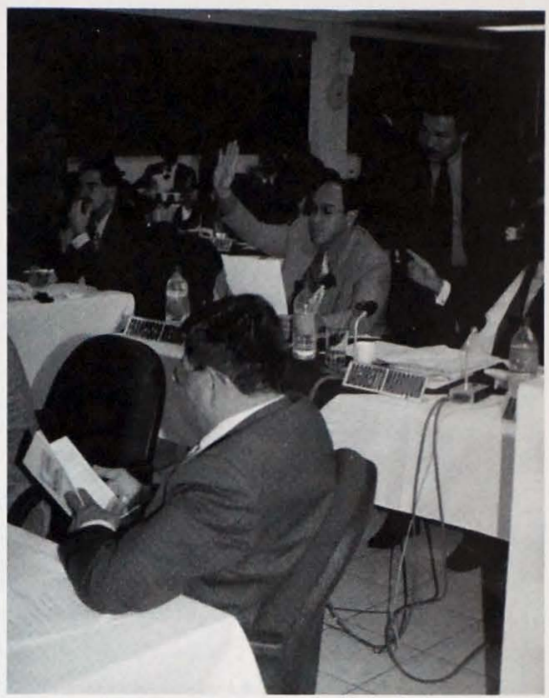

blemente la llegada del partido de oposición lastimaba la soberbia del partido de gobierno y su imagen externa. De cualquier forma es un funesto precedente para una incipiente democracia.

\section{El papel de los partidos políticos}

Tos partidos políticos son en reoría los legítimos representantes de los distintos sectores de la población. En nuestro país difícilmente puede decirse que representan a alguien, como no sea a grupos pequeños de intereses muy identificados con las cúpulas dirigenciales de cada partido. En otras palabras la impresión popular es que no representan a nadie, y que se encuentran tan alejados de las necesidades y problemas de la población que no son más que figuras decorativas, males necesarios de un sistema republicano que no satisface el papel que en teoría les compete.

La desconfianza y la frustración frente a los partidos políticos es cada vez más evidente y lo evidencian los altos indices de ausentismo durante las últimas elecciones. Luego, la agobiante sensación de su permanente esterilidad y su manifiesta incapacidad para encontrar y descubrir la razón última de su existencia en el sistema democrático, como es la consecución del bien común. La función principal del 
partido es manecner abicrtas y despejadas las víus de comunicación enere la población y los dirigentes, sirviendo de orientadores enere las necesidades populares y los legíimos incereses de la función publica. Cuando dicho papel se encuenera ausente, l. inconformidad social ciende a manifestarse por diferentes vias incluidas las manifestaciones populares de expresión violentia.

Un análisis objecivo del papel actual de los parridos políticos en la vida salvadoreña no deja de ser desalentador. Posiblemente con excepción del parrido ARENA, el resto de partidos muestran una evidente inconsistencia entre sus postulados y la praxis: a tal grado que los electores se sienecon confundidos y frustrados. La cuestión básica hacia el futuro es que en la medida que se acentuc la inoperarividad de los partidos políticos, la organización de grupos de presión para manifestarse por otras vias es bastante probable; lo que es un indicador de que el sistema llamado democratico no funciona y se requieren nuevas formas de expresión colectiva.

\section{El papel del FMLN}

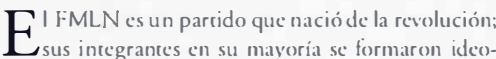
lógicamente en los campos de batalla en un esfuerzo bélico que se prolongó por más de diez años. Sus seguidores, acrivos y pasivos, en las ciudades y el campo, estuvieron motivados por una definida línea de lucha ancisistema y una entrega personal sin antecedentes en la historia política del pais durante los tilumos cincuenta años. Los antecedentes históricos de lucha popular podrian ser únicamente la gesta de Anastasio Aquino en 1833 y la llamada revolución campesina de la década de los treinea.

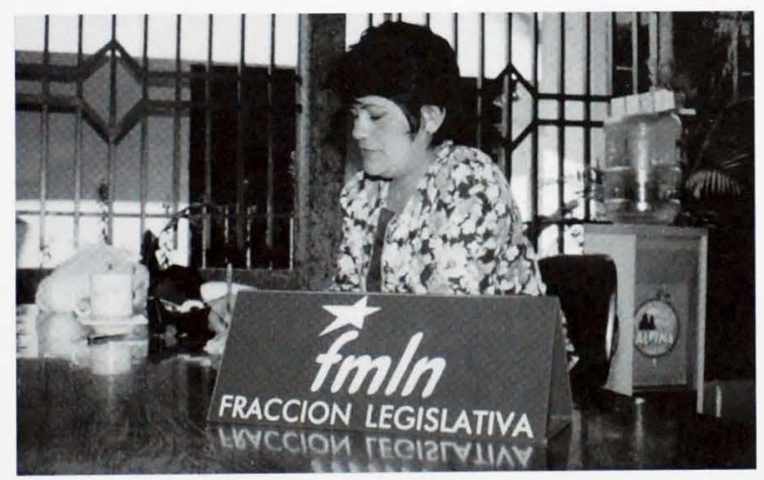

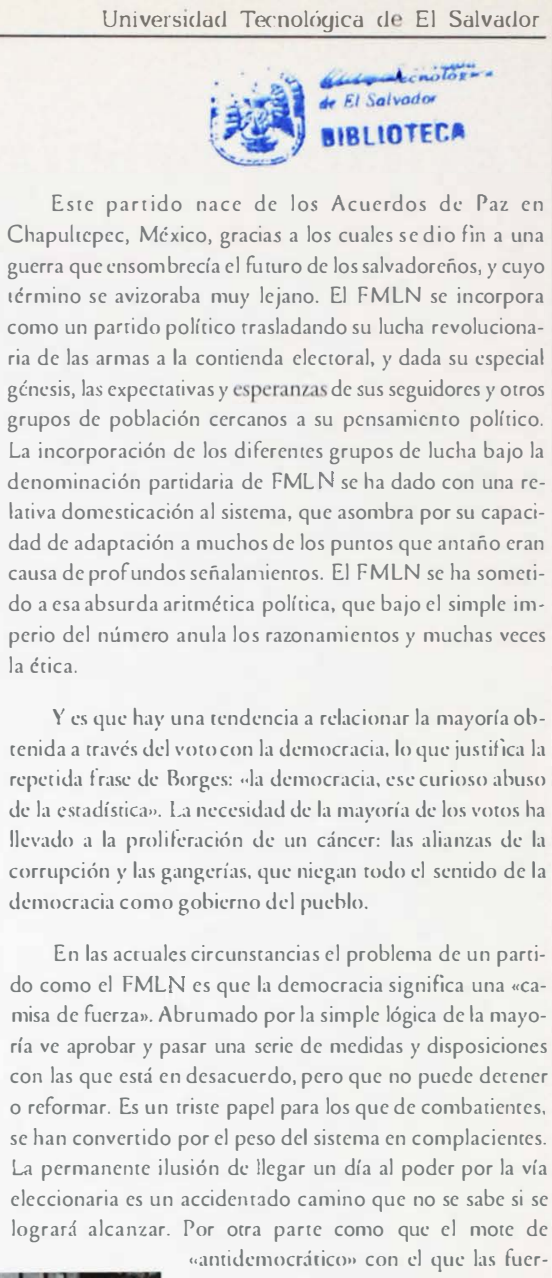
la érica. democracia como gobierno del pucblo. zas de la derecha calitican a codo aquel que no esté de acuerdo a sus intereses, se ha convertido en una mordaza y en un obstáculo para otras iniciativas.

El papel pasivo del FMLN riene desconcertados a muchos de aquellos que depositaron su confianza en el movimienro, contradictoriamente en pos de un proceso democrárico más transparente. No está de más recomendar a los dirigentes partidarios una profunda revisión de sus actuaciones y contribuciones en la búsqueda de los objerivos primigenios de su lucha. 